\title{
HANDWRITTEN CHARACTER RECOGNITION USING STRUCTURAL SHAPE DECOMPOSITION
}

\author{
Abdullah A. Al-Shaher ${ }^{1}$ and Edwin R. Hancock ${ }^{2}$ \\ ${ }^{1}$ Department of Computer and Information Systems, College of Business \\ Studies, Public Authority for Applied Education and Training, Kuwait \\ ${ }^{2}$ Department of Computer Science, University of York, York, United Kingdom
}

\begin{abstract}
This paper presents a statistical framework for recognising $2 D$ shapes which are represented as an arrangement of curves or strokes. The approach is a hierarchical one which mixes geometric and symbolic information in a three-layer architecture. Each curve primitive is represented using a point-distribution model which describes how its shape varies over a set of training data. We assign stroke labels to the primitives and these indicate to which class they belong. Shapes are decomposed into an arrangement of primitives and the global shape representation has two components. The first of these is a second point distribution model that is used to represent the geometric arrangement of the curve centre-points. The second component is a string of stroke labels that represents the symbolic arrangement of strokes. Hence each shape can be represented by a set of centre-point deformation parameters and a dictionary of permissible stroke label configurations. The hierarchy is a two-level architecture in which the curve models reside at the nonterminal lower level of the tree. The top level represents the curve arrangements allowed by the dictionary of permissible stroke combinations. The aim in recognition is to minimise the cross entropy between the probability distributions for geometric alignment errors and curve label errors. We show how the stroke parameters, shape-alignment parameters and stroke labels may be recovered by applying the expectation maximization EM algorithm to the utility measure. We apply the resulting shape-recognition method to Arabic character recognition.
\end{abstract}

\section{KEYWORDS}

point distribution models, expectation maximization algorithm, discrete relaxation, hierarchical mixture of experts, Arabic scripts, handwritten characters

\section{INTRODUCTION}

The analysis and recognition of curved shapes has attracted considerable attention in the computer vision literature. Current work is nicely exemplified by point distribution models [1] and shape-contexts [2]. However, both of these methods are based on global shape-descriptors. This is potentially a limitation since a new model must be acquired for each class of shape and this is an inefficient process. An alternative and potentially more flexible route is to use a structural approach to the problem, in which shapes are decomposed into arrangements of Dhinaharan Nagamalai et al. (Eds) : CoSIT, SIGL, AIAPP, CYBI, CRIS, SEC, DMA - 2017

pp. 39- 47, 2017. (C) CS \& IT-CSCP 2017

DOI : $10.5121 /$ csit.2017.70405 
primitives. This idea was central to the work of Marr [3]. Shape learning may then be decomposed into a two-stage process. The first stage is to acquire a models of the variability is the distinct primitives. The second stage is to learn the arrangements of the primitives corresponding to different shape classes.

Although this structural approach has found widespread use in the character recognition community, it has not proved popular in the computer vision domain. The reason for this is that the segmentation of shapes into stable primitives has proved to be an elusive problem. Hence, there is a considerable literature on curve polygonalisation, segmentation and grouping. However, one of the reasons that the structural approach has proved fragile is that it has been approached using geometric rather than statistical methods. Hence, the models learned and the recognition results obtained are highly sensitive to segmentation error. In order to overcome these problems, in this paper we explore the use of probabilistic framework for recognition.

We focus on the problem of developing hierarchical shape models for handwritten Arabic characters. These characters are decomposed into concave or convex strokes. Our statistical learning architecture is reminiscent of the hierarchical mixture of experts algorithm. This is a variant of the expectation maximisation algorithm, which can deal with hierarchically structured models. The method was first introduced in 1994 by Jordan and Jacobs [4]. In its simplest form the method models data using a doubly nested mixture model. At the top layer the mixture is over a set of distinct object classes. This is sometimes referred to as the gating layer. At the lower level, the objects are represented as a mixture over object subclasses. These sub-classes feed into the gating later with predetermined weights. The parameters of the architecture reside in the sublayer, which is frequently represented using a Gaussian mixture model. The hierarchical mixture of experts algorithm provides a means of learning both the gating weights and the parameters of the Gaussian mixture model.

Here our structural decomposition of 2D shapes is a hierarchical one which mixes geometric and symbolic information. The hierarchy has a two-layer architecture. At the bottom layer we have strokes or curve primitives. These fall into different classes. For each class the curve primitive is represented using a point-distribution model [5] which describes how its shape varies over a set of training data. We assign stroke labels to the primitives to distinguish their class identity. At the top level of the hierarchy, shapes are represented as an arrangement of primitives. The representation of the arrangement of primitives has two components. The first of these is a second point distribution model that is used to represent how the arrangement of the primitive centrepoints varies over the training data. The second component is a dictionary of configurations of stroke labels that represents the arrangements of strokes at a symbolic level. Recognition hence involves assigning stroke symbols to curves primitives, and recovering both stroke and shape deformation parameters. We present a probabilistic framework which can be for the purposes of shape-recognition in the hierarchy. We apply the resulting shape-recognition method to Arabic character recognition.

\section{SHAPE REPRESENTATION}

We are concerned with recognising a shape $W=\{\underset{w}{w}, \ldots, \underset{w}{w}\}$ which consists of a set of $p$ ordered but unlabelled landmark points with $2 \mathrm{D}$ co-ordinate vectors $\underset{w_{1}}{\boldsymbol{\mu}}, \ldots, \underset{w_{p}}{\boldsymbol{\mu}}$. The shape is assumed to be segmented into a set of $K$ non-overlapping strokes. Each stroke consists of a set 
of consecutive landmark points. The set of points belonging to the stroke indexed $k$ is $S_{k}$. For each stroke, we compute the mean position

$$
\stackrel{\rho}{c_{k}}=\frac{1}{\left|S_{k}\right|} \sum_{i \in S_{k}}^{\rho} \stackrel{\rho}{w}_{i}
$$

The geometry of stroke arrangement is captured by the set of mean position vectors $C=\left\{\stackrel{\mu}{c_{1}, \ldots . . c_{K}}\right\}$.

Our hierarchical model of the characters uses both geometric and symbolic representations of the shapes. The models are constructed from training data. Each training pattern consists of a set of landmark points that are segmented into strokes. We commence by specifying the symbolic components of the representation. Each training pattern is assigned to shape class and each component stroke is assigned to stroke class. The set of shape-labels is $\Omega_{c}$ and the set of stroke labels is $\Omega_{s}$. The symbolic structure of each shape is represented a permissible arrangement of stroke-labels. For shapes of class $\omega \in \Omega_{c}$ the permissible arrangement of strokes is denoted by

$$
\Lambda_{\omega}=<\lambda_{1}^{\omega}, \lambda_{2}^{\omega}, \ldots>
$$

We model the geometry of the strokes and stroke-centre arrangements using point distribution models. To capture the shape variations, we use training data. The data consists of a set of shapes which have been segmented into strokes. Let the $t^{\text {th }}$ training pattern consist of the set of $p$ landmark co-ordinate vectors $X^{t}=\left\{f_{1}, \ldots \ldots, x_{p}\right\}$. Each training pattern is segmented into strokes. For the training pattern indexed $t$ there are $K_{t}$ strokes and the index set of the points belonging to the $k^{\text {th }}$ stroke is $S_{k}^{t}$. To construct the point distribution model for the strokes and stroke-centre arrangements, we convert the point co-ordinates into long-vectors. For the training pattern indexed $t$, the long-vector of stroke centres is $X_{t}=\left(\left(c_{1}^{t}\right)^{T},\left(p_{2}\right)^{T}, \ldots,\left(p_{t}\right)^{T}\right)^{T}$. Similarly for the stroke indexed $k$ in the training pattern indexed $t$, the long-vector of co-ordinates is denoted by $z_{t, k}$. For examples shapes belonging to the class $\omega$, to construct the stroke-centre point distribution model we need to first compute the mean long vector

$$
Y_{\omega}=\frac{1}{\left|T_{\omega}\right|} \sum_{t \in T_{\omega}} X_{t}
$$

where $T_{\omega}$ is the set of index patterns and the associated covariance matrix

$$
\Sigma_{\omega}=\frac{1}{\left|T_{\omega}\right|} \sum_{t \in T_{\omega}}\left(X_{t}-Y_{\omega}\right)\left(X_{t}-Y_{\omega}\right)^{T}
$$


The eigenmodes of the stroke-centre covariance matrix are used to construct the point-distribution model. First, the eigenvalues $e$ of the stroke covariance matrix are found by solving the eigenvalue equation $\left|\Sigma_{\omega}-e^{\omega} I\right|=0$ where $I$ is the $2 L \times 2 L$ identity matrix. The eigen-vector $\phi_{i}$ corresponding to the eigenvalue $e_{i}^{\omega}$ is found by solving the eigenvector equation $\Sigma \phi_{i}^{\omega}=e_{i}^{\omega} \phi_{i}^{\omega}$. According to Cootes and Taylor [6], the landmark points are allowed to undergo displacements relative to the mean-shape in directions defined by the eigenvectors of the covariance matrix $\Sigma_{\omega}$. To compute the set of possible displacement directions, the $M$ most significant eigenvectors are ordered according to the magnitudes of their corresponding eigenvalues to form the matrix of column-vectors $\Phi_{\omega}=\left(\phi_{1}^{\omega}\left|\phi_{2}^{\omega}\right| \ldots \mid \phi_{M}^{\omega}\right)$, where $e_{1}^{\omega}, e_{2}^{\omega}, \ldots . ., e_{M}^{\omega}$ is the order of the magnitudes of the eigenvectors. The landmark points are allowed to move in a direction which is a linear combination of the eigenvectors. The updated landmark positions are given by $\hat{X}=Y_{\omega}+\Phi_{\omega} \gamma_{\omega}$, where $\gamma_{\omega}$ is a vector of modal co-efficients. This vector represents the free-parameters of the global shape-model.

This procedure may be repeated to construct a point distribution model for each stroke class. The set of long vectors for strokes of class $\lambda$ is $T_{\lambda}=\left\{Z_{t, k} \mid \lambda_{k}^{t}=\lambda\right\}$. The mean and covariance matrix for this set of long-vectors are denoted by $Y_{\lambda}$ and $\Sigma_{\lambda}$ and the associated modal matrix is $\Phi_{\lambda}$. The point distribution model for the stroke landmark points is $\hat{Z}=Y_{\lambda}+\Phi_{\lambda} \gamma_{\lambda}$.

We have recently described how a mixture of point-distribution models may be fitted to samples of shapes. The method is based on the EM algorithm and can be used to learn point distribution models for both the stroke and shape classes in an unsupervised manner. We have used this method to learn the mean shapes and the modal matrices for the strokes. More details of the method are found in [7].

\section{HiERARCHICAL ARCHITECTURE}

With the stroke and shape point distribution models to hand, our recognition method proceeds in a hierarchical manner. To commence, we make maximum likelihood estimates of the best-fit parameters of each stroke-model to each set of stroke-points. The best-fit parameters $\gamma_{\lambda}^{k}$ of the stroke-model with class-label $\lambda$ to the set of points constituting the stroke indexed $k$ is

$$
\gamma_{\lambda}^{k}=\arg \max _{\gamma} p\left(z_{k} \mid \Phi_{\lambda}, \gamma\right)
$$

We use the best-fit parameters to assign a label to each stroke. The label is that which has maximum a posteriori probability given the stroke parameters. The label assigned to the stroke indexed $k$ is 


$$
l_{k}=\arg \max _{\lambda \in \Omega_{s}} P\left(l \mid z_{k}, \gamma_{\lambda}, \Phi_{\lambda}\right)
$$

In practice, we assume that the fit error residuals follow a Gaussian distribution. As a result, the class label is that associated with the minimum squared error. This process is repeated for each stroke in turn. The class identity of the set of strokes is summarised the string of assigned strokelabels

$$
L=<l_{1}, l_{2}, \ldots .>
$$

Hence, the input layer is initialised using maximum likelihood stroke parameters and maximum a posteriori probability stroke labels.

The shape-layer takes this information as input. The goal of computation in this second layer is to refine the configuration of stroke labels using global constraints on the arrangement of strokes to form consistent shapes. The constraints come from both geometric and symbolic sources. The geometric constraints are provided by the fit of a stroke-centre point distribution model. The symbolic constraints are provide by a dictionary of permissible stroke-label strings for different shapes.

The parameters of the stroke-centre point distribution model are found using the EM algorithm [8]. Here we borrow ideas from the hierarchical mixture of experts algorithm, and pose the recovery of parameters as that of maximising a gated expected log-likelihood function for the distribution of stroke-centre alignment errors $p\left(X \mid \Phi_{\omega}, \Gamma_{\omega}\right)$. The likelihood function is gated by two sets of probabilities. The first of these are the a posteriori probabilities $P\left(\lambda_{k}^{\omega} \mid z_{k}, \gamma_{\lambda_{k}^{\omega}}, \Phi_{\lambda_{k}^{\omega}}\right)$ of the individual strokes. The second are the conditional probabilities $P\left(L \mid \Lambda_{\omega}\right)$ of the assigned stroke-label string given the dictionary of permissible configurations for shapes of class $\omega$. The expected log-likelihood function is given by

$$
L=\sum_{\omega \in \Omega_{c}} P\left(L \mid \Lambda_{\omega}\right)\left\{\prod_{k} P\left(\lambda_{k}^{\omega} \mid z_{k}, \gamma_{\lambda_{k}^{\omega}}, \Phi_{\lambda_{k}^{\omega}}\right)\right\} \ln p\left(X \mid \Phi_{\omega}, \Gamma_{\omega}\right)
$$

The optimal set of stroke-centre alignment parameters satisfies the condition

$$
\Gamma_{\omega}^{*}=\arg \max _{\Gamma} P\left(L \mid \Lambda_{\omega}\right)\left\{\prod_{k} P\left(\lambda_{k}^{\omega} \mid z_{k}, \gamma_{\lambda_{k}^{\omega}}, \Phi_{\lambda_{k}^{\omega}}\right)\right\} \ln p\left(X \mid \Phi_{\omega}, \Gamma_{\omega}\right)
$$

From the maximum likelihood alignment parameters we identify the shape-class of maximum $a$ posteriori probability. The class is the one for which

$$
\omega^{*}=\arg \max _{\omega \in \Omega_{c}} P\left(\omega \mid X, \Phi_{\omega}, \Gamma_{\omega}^{*}\right)
$$


The class identity of the maximum a posteriori probability shape is passed back to the strokelayer of the architecture. The stroke labels can then be refined in the light of the consistent assignments for the stroke-label configuration associated with the shape-class $\omega$.

$$
l_{k}=\arg \max _{\lambda \in \Omega_{s}} P\left(\lambda \mid z_{k}, \gamma_{l}^{k}, \Phi_{\lambda}\right) P\left(L(\lambda, k) \mid \Lambda_{\omega}\right)
$$

Finally, the maximum likelihood parameters for the strokes are refined

$$
\left.\gamma_{k}=\arg \max _{\gamma} p\left(\xi_{k} \mid \Phi_{l_{k}}, \gamma, \Gamma_{\omega}^{*}\right)\right)
$$

These labels are passed to the shape-layer and the process is iterated to convergence.

\section{MODELS}

In this section we describe the probability distributions used to model the point-distribution alignment process and the symbol assignment process.

\subsection{Point-Set Alignment}

To develop a useful alignment algorithm we require a model for the measurement process. Here we assume that the observed position vectors, i.e. $\boldsymbol{w}_{i}$ are derived from the model points through a Gaussian error process. According to our Gaussian model of the alignment errors,

$$
p\left(z_{k} \mid \Phi_{\lambda}, \gamma_{\lambda}\right)=12 \pi \sigma \exp \left[-12 \sigma^{2}\left(\underline{z}_{k}-Y_{\omega}-\Phi_{\omega} \gamma_{\lambda}\right)^{T}\left(\xi_{k}-Y_{\omega}-\Phi_{\omega} \gamma_{\lambda}\right)\right]
$$

where $\sigma^{2}$ is the variance of the point-position errors which for simplicity are assumed to be isotropic. The maximum likelihood parameter vector is given by

$$
\gamma_{\lambda}^{k}=\frac{1}{2}\left(\Phi_{\omega}^{T} \Phi_{\omega}\right)^{-1}\left(\Phi_{\omega}+\Phi_{\omega}^{T}\right)\left(\stackrel{\rho}{k}_{k}-Y_{\omega}\right)
$$

A similar procedure may be applied to estimate the parameters of the stroke centre point distribution model.

\subsection{Label Assignment}

The distribution of label errors is modelled using the method developed by Hancock and Kittler [9]. To measure the degree of error we measure the Hamming distance between the assigned string of labels $L$ and the dictionary item $\Lambda$. The Hamming distance is given by

$$
H\left(L, \Lambda_{\omega}\right)=\sum_{i=1}^{K} \delta_{l_{i}, \lambda_{i}^{\omega}}
$$

where $\delta$ is the DiracDelta function. With the Hamming distance to hand, the probability of the assigned string of labels $L$ given the dictionary item $\Lambda$ is

$$
P\left(L \mid \Lambda_{\omega}\right)=K_{p} \exp \left[-k_{p} H\left(L, \Lambda_{\omega}\right)\right]
$$




$$
\text { where } K_{p}=(1-p)^{K} \text { and } k_{p}=\ln \frac{1-p}{p}
$$

are constants determined by the label-error probability $p$.

\section{EXPERIMENT}

We have evaluated our approach on sets of Arabic characters. Figure 1 shows some of the data used for the purpose of learning and recognition. In total we use, 18 distinct classes of Arabic characters and for each class there are 200 samples. The landmarks are placed uniformly along the length of the characters. The top row shows the example pattern used for training a representative set of shape-classes. The second row of the figure shows the configuration of mean strokes for each class. Finally, the third row shows the stroke centre-points.
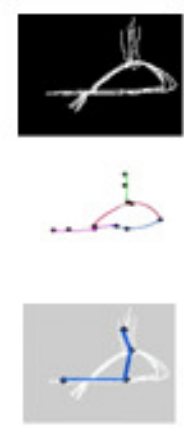
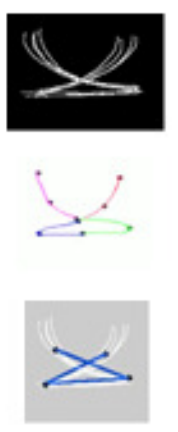
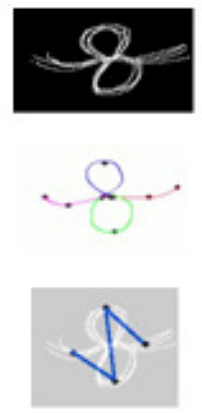
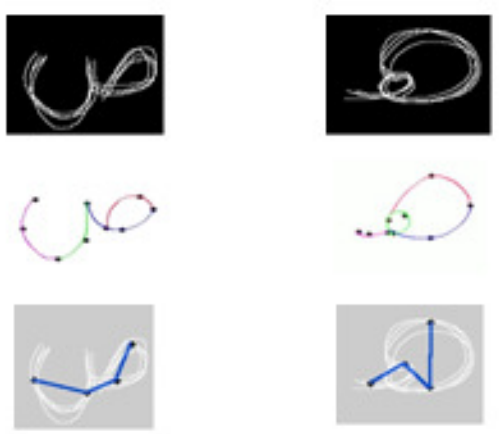

Figure 1: Raw 1 shows sample training sets. Raw 2 shows stroke mean shapes, Raw 3 shows stroke arrangements

Table 1 shows the results for a series of recognition experiments. The top raw of the table shows the character shape class. For each shape-class, we have used 200 test patterns to evaluate the recognition performance. These patterns are separate from the data used in training. The raws of the table compare the recognition results obtained using a global point-distribution model to represent the character shape and using the stroke decomposition model described in this paper. We list the number of correctly identified patterns. In the case of the global PDM, the average recognition accuracy is $93.2 \%$ over the different character classes. However, in the case of the stroke decomposition method the accuracy is $97 \%$. Hence, the new method offers a performance gain of some $5 \%$.

Table 1. Recognition Rate for shape-classes ( Full Character, Stroke-based arrangements )

\begin{tabular}{|c|c|c|c|c|c|c|c|c|c|c|c|c|}
\hline Shape & $\varepsilon$ & $\ddots$ & $\searrow$ & $C$ & $J$ & $\bigcup$ & 9 & $\ddots$ & 6 & -8 & $b$ & $\cup$ \\
\hline Character & 190 & 195 & 192 & 190 & 193 & 177 & 184 & 183 & 178 & 186 & 187 & 182 \\
\hline Stroke & 197 & 198 & 197 & 199 & 198 & 189 & 196 & 192 & 194 & 190 & 193 & 188 \\
\hline
\end{tabular}


Figure 2 examines the iterative qualities of the method. Here we plot the a posteriori class probability as a function of iteration number when recognition of characters of a specific class is attempted. The different curves are for different classes. The plot shows that the method converges in about six iterations and that the probabilities of the subdominant classes tend to zero.

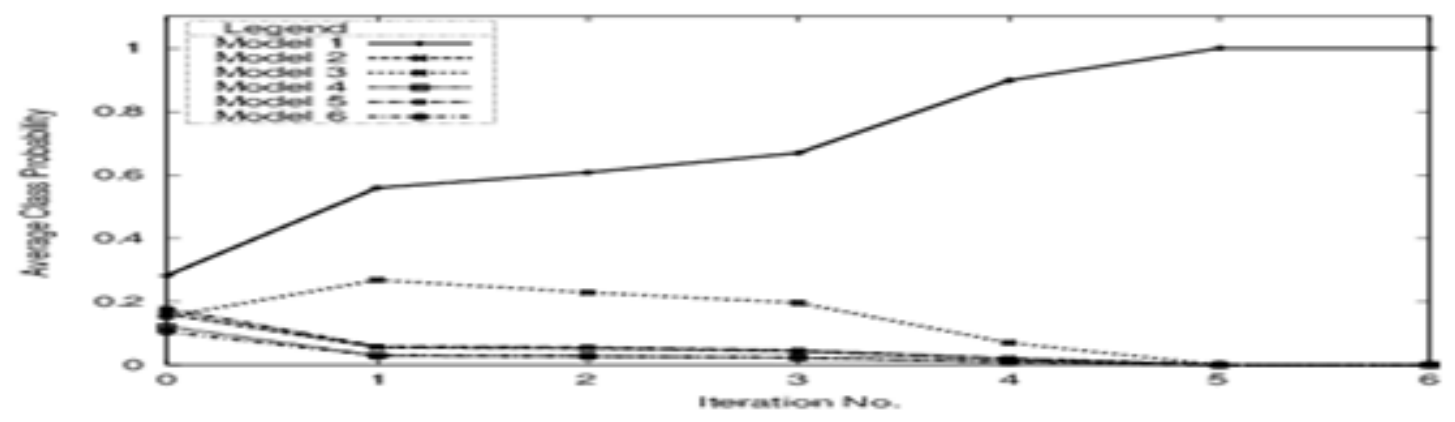

Figure 1. Alignment convergence rate as a function per iteration number

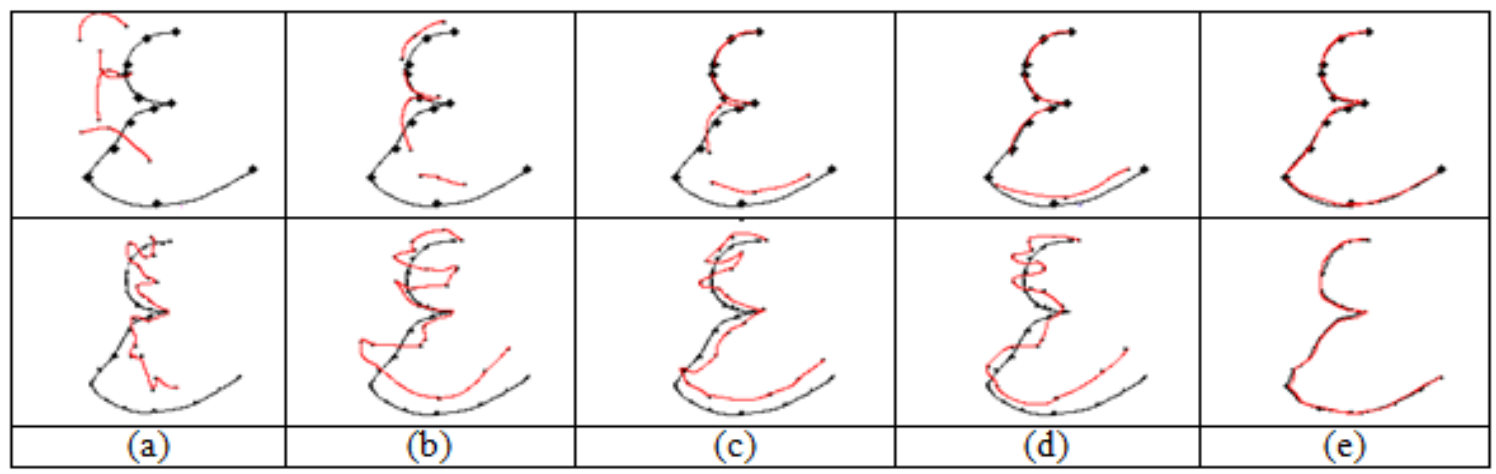

Figure 2. Alignment. First raw shows hierarchical strokes:(a)iteration 1, (b)iteration 2, (c)iteration 3, (d)iteration 4, (e)iteration 5. Second raw represents character models:(a)iteration 1, (b)iteration 2, (c)iteration 3, (d)iteration 4, (d)iteration 8

Figure 3 compares the fitting of the stroke model (top row) and a global PDM (bottom row) with iteration number. It is clear that the results obtained with the stroke model are better than those obtained with the global PDM, which develops erratic local deformations. Finally, we demonstrate in Figure 4 comparison of stroke decomposition and character with respect to recognition rate as a function of point position error. Stroke decomposition methods shows a better performance when points are moved randomly away of their original location.

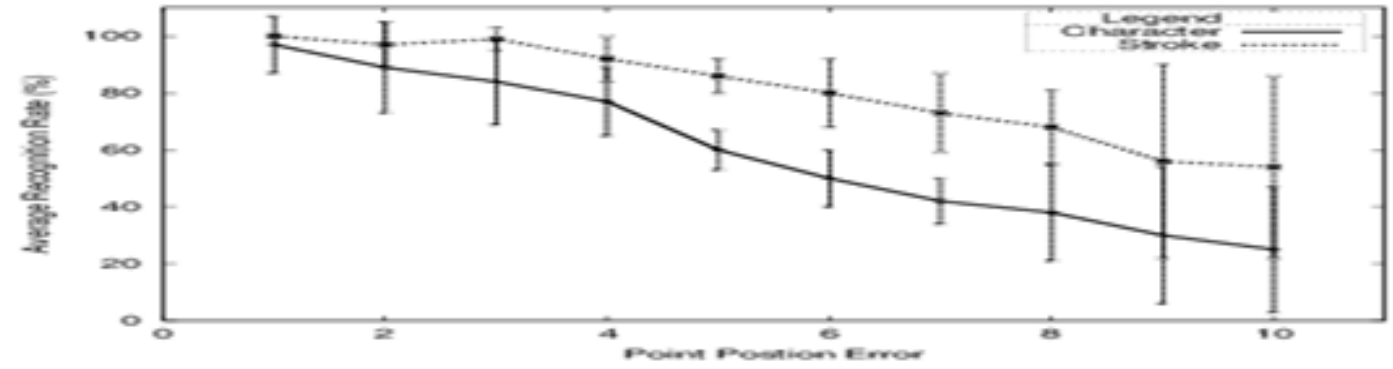

Figure 4. Recognition rate with respect to random point position 


\section{CONCLUSION}

In This Paper, we have described a hierarchical probabilistic framework for shape recognition via stroke decomposition. The structural component of the method is represented using symbolic dictionaries, while geometric component is represented using Point Distribution Models. Experiments on Arabic character recognition reveal that the method offers advantages over a global PDM.

\section{REFERENCES}

[1] T. Cootes, C. Taylor, D. Cooper and J. Graham, "Active Shape Models-Their Training and Application," Computer Vision and Image Understanding, vol. 61, no. 1, pp. 38-59, 1995.

[2] S. Belongie, J. Malik and a. J. Puzicha, "Shape matching and object recognition using shape contexts," IEEE Transactions on PAMI, vol. 24, no. 24, pp. 509-522, 2002.

[3] D. C. Marr, Vision: a computational investigation into the human representation and processing of visual information, San Francisco: Freeman, 1982.

[4] M. Jordan and R. Jacobs, "Hierarchical mixtures of experts and the em algorithm," Neural Computation, vol. 6, pp. 181-214, 1994.

[5] C. T. and T. C., "A mixture models for representing shape variation," Image and Vision Computing, vol. 17, pp. 403-409, 1999.

[6] C. T. and T. C., "Combining point distribution models with shape models based on finite element analysis," Image and Vision Computing, vol. 13, no. 5, pp. 403-409, 1995.

[7] A. A. Al-Shaher and E. R. Hancock, "Linear shape recognition with mixtures of point distribution models," in SSPR, Windsor, Canada, 2002.

[8] A. Dempster, L. N. and R. D., "Maximum likelihood from incomplete data via the em algorithm," Journal of Royal Statistical Soc. Ser., vol. 39, pp. 1-38, 1977.

[9] E. R. Hancock and J. Kittler, "Discrete relaxation," Pattern Recognition, vol. 23, no. 7, pp. 711-733, 1990. 